\title{
Evaluation of osteopenia and osteoporosis in younger breast cancer survivors compared with cancer-free women: a prospective cohort study
}

Cody Ramin ${ }^{1}$, Betty J. May', Richard B. S. Roden², Mikiaila M. Orellana', Brenna C. Hogan', Michelle S. McCullough', Dana Petry ${ }^{3}$, Deborah K. Armstrong ${ }^{2}$ and Kala Visvanathan ${ }^{1,2,3^{*}}$ (D)

\begin{abstract}
Background: Osteoporosis, an indicator of significant bone loss, has been consistently reported among older breast cancer survivors. Data are limited on the incidence of osteopenia, an earlier indicator of bone loss, and osteoporosis in younger breast cancer survivors compared with cancer-free women.

Methods: We prospectively examined bone loss in 211 breast cancer survivors (mean age at breast cancer diagnosis $=47$ years) compared with 567 cancer-free women in the same cohort with familial risk for breast cancer. Multivariable-adjusted Cox proportional hazards models were used to estimate HRs and $95 \% \mathrm{Cls}$ of osteopenia and/or osteoporosis incidence based on physician diagnosis.
\end{abstract}

Results: During a mean follow-up of 5.8 years, $66 \%$ of breast cancer survivors and $53 \%$ of cancer-free women reported having a bone density examination, and 112 incident cases of osteopenia and/or osteoporosis were identified. Breast cancer survivors had a $68 \%$ higher risk of osteopenia and osteoporosis compared to cancer-free women ( $\mathrm{HR}=1.68,95 \% \mathrm{Cl}=1.12-2.50)$. The association was stronger among recent survivors after only 2 years of follow-up ( $\mathrm{HR}=2.74,95 \% \mathrm{Cl}=1.37-5.47)$. A higher risk of osteopenia and osteoporosis was also observed among survivors aged $\leq 50$ years, estrogen receptor-positive tumors, and those treated with aromatase inhibitors alone or chemotherapy plus any hormone therapy relative to cancer-free women.

Conclusions: Younger breast cancer survivors are at higher risk for osteopenia and osteoporosis compared to cancer-free women. Studies are needed to determine effective approaches to minimize bone loss in this population.

Keywords: Osteopenia, Osteoporosis, Bone loss, Breast cancer survivors, Cancer-free women

\section{Introduction}

Osteopenia and osteoporosis, both systemic skeletal conditions associated with varying degrees of bone loss, are prevalent among postmenopausal breast cancer survivors, with prior reports of up to $80 \%$ experiencing loss in bone density [1]. Untreated bone loss can lead to significant morbidity due to pain and fractures, as well as to death [2]. Osteopenia is diagnosed among individuals

\footnotetext{
* Correspondence: kvisvan1@jhu.edu

'Department of Epidemiology, Johns Hopkins Bloomberg School of Public Health, Baltimore, MD 21205, USA

${ }^{2}$ Johns Hopkins Sidney Kimmel Comprehensive Cancer Center, Baltimore, MD, USA

Full list of author information is available at the end of the article
}

with lower-than-average bone density, while osteoporosis is characterized by both low bone density and architectural deterioration of bone tissue [3]. Among breast cancer survivors, cancer-related risk factors for osteopenia and osteoporosis include both treatment and premature menopause [4]. Importantly, the excess risk of osteopenia and osteoporosis among breast cancer survivors, particularly those of a younger age, relative to their cancer-free peers remains unknown.

Osteopenia and osteoporosis are also prevalent in the general population. Among women aged $\geq 50$ years in the United States, approximately $15.4 \%$ have osteoporosis and 51.4\% have low bone density [5]. Furthermore, 
it is estimated that 1 in 2 women will be at risk for an osteoporosis-related fracture during their lifetime $[2,6]$. Among cancer-free women, loss in bone density is associated with advancing age, menopause-induced estrogen deficiency, low body weight, lack of physical activity, excess alcohol consumption, family history of bone fracture, cigarette smoking, low calcium intake, and vitamin D deficiency $[4,7]$. Loss of bone density in cancer survivors could be due to similar risk factors in addition to treatment-related effects. By comparing cancer survivors with cancer-free individuals, these risk factors can be differentiated.

Few epidemiologic studies have examined osteopenia and osteoporosis in breast cancer survivors relative to cancer-free women within the same cohort [8-10]. One prior study reported significantly lower levels of bone mineral density [8], the gold standard for assessing bone loss, and two other previous studies observed an increased risk of osteopenia and osteoporosis $[9,10]$ compared with cancer-free women. These studies were primarily conducted among older and long-term breast cancer survivors and did not differentiate based on tumor subtypes and detailed treatment regimens. One reason for the paucity of studies among younger breast cancer survivors is likely the challenge of identifying a comparable cancerfree group, because young cancer-free women do not routinely undergo assessment for bone health. Fortunately, we found this not to be the case in women with familial breast cancer risk, and we were therefore able to prospectively examine the risk of osteopenia and osteoporosis in a familial risk cohort known as the Breast and Ovarian Surveillance Service (BOSS) study.

\section{Methods}

\section{Study population}

The BOSS study is an ongoing prospective cohort study that includes women and men with familial risk for breast and/or ovarian cancer [11]. Participants were enrolled from 2005 to 2013 primarily from the Clinical Cancer Genetics Program at The Johns Hopkins Sidney Kimmel Comprehensive Cancer Center in Baltimore, MD, USA. Participants were aged $\geq 18$ years with either (1) a family history of breast and/or ovarian cancer, (2) a documented BRCA1/2 mutation, (3) a diagnosis of breast cancer at $\leq 40$ years of age without a family history of breast cancer, or (4) a diagnosis of ovarian cancer at any age without a family history of ovarian cancer. Participants completed a baseline questionnaire so that information could be collected on a variety of demographic, lifestyle, and health-related factors, including detailed information on medical history and breast cancer treatment. Subsequent follow-up questionnaires have been completed every 3-4 years thereafter ( $>92 \%$ have completed at least one follow-up questionnaire). Completion of the second and third follow-up questionnaires is ongoing.

For the present prospective analysis, women were included if they completed a baseline questionnaire and at least one follow-up questionnaire through September 30, $2017(n=1173)$. Women with a physician diagnosis of osteopenia or osteoporosis at baseline $(n=272$ total; $n=$ 174 with osteopenia only; $n=46$ osteoporosis only) or bisphosphonate use at baseline $(n=5)$ were excluded. We further excluded women with missing responses for osteopenia or osteoporosis on baseline $(n=1)$ or followup $(n=5)$ questionnaires. For this analysis, breast cancer survivors were defined as women diagnosed with breast cancer (ductal carcinoma in situ [stage 0] or stages I-III breast cancer) within 5 years prior to enrollment. The comparison group was restricted to women with no prior history of cancer at baseline except nonmelanoma skin cancer or cervical carcinoma in situ. After these exclusions, 778 women (211 breast cancer survivors and 567 cancer-free) became our analytic study population.

\section{Exposure assessment}

Cancer diagnoses were self-reported at enrollment, and pathology records were reviewed to confirm all diagnoses (by International Classification of Diseases Codes, Tenth Revision: invasive breast cancer [C50]; ductal carcinoma in situ [D05.1], and lobular carcinoma in situ [D05.0]) as well as stage and hormone receptor status (estrogen receptor [ER] /progesterone receptor [PR] and human epidermal growth factor receptor 2 [HER2]). Breast cancer treatment was reported in baseline questionnaires, and details were confirmed by medical record review (96\% confirmed). Treatment information included surgery (none, lumpectomy, mastectomy) and adjuvant therapy (chemotherapy, radiation, and hormone therapy). Detailed information on type of chemotherapy and hormone therapy was also collected. We classified cancer treatment into mutually exclusive categories of surgery only, hormone therapy alone, chemotherapy alone, and chemotherapy plus hormone therapy. Hormone therapy was further classified as tamoxifen or aromatase inhibitor use.

\section{Outcome assessment}

Osteopenia and osteoporosis diagnoses were ascertained in baseline and follow-up questionnaires. In each questionnaire, participants were asked to indicate whether they had received a physician's diagnosis of osteopenia or osteoporosis and the date of that diagnosis. Incident cases of osteopenia and osteoporosis were identified on follow-up questionnaires. Our outcome of interest was a composite outcome that included incident osteopenia (i.e., low bone mass) and/or osteoporosis. Participants also reported whether they had ever had a bone density 
examination and the year of examination on both baseline and follow-up questionnaires.

\section{Ascertainment of covariates}

Information on covariates (age, race, education level, menopausal status, age at menopause, oophorectomy at a young age, body mass index [BMI], physical activity based on metabolic equivalents of task [METs] per week, alcohol intake, smoking status, hormone replacement therapy [HRT] use, current bisphosphonate use, vitamin D supplement use, and calcium supplement use) was available from the baseline questionnaire. Bilateral oophorectomy at a young age was defined as both ovaries removed prior to age 45 years and based on self-report. We calculated age at bilateral oophorectomy from the date that the second ovary was removed. Medical procedures and screening examinations, including mammograms, pap smears, sigmoidoscopy, and colonoscopy, were also reported on both baseline and follow-up questionnaires.

\section{Statistical analysis}

Baseline characteristics of breast cancer survivors and cancer-free women were compared with frequency distributions for categorical variables and means (SDs) for continuous variables. We used Cox proportional hazards models with age as the time scale to calculate HRs and 95\% CIs. Women contributed person-time from the completion date of the baseline questionnaire to the date of osteopenia or osteoporosis diagnosis or until the end of the last follow-up through September 30, 2017, whichever occurred first. The proportional hazards assumption was assessed with log-log survival plots and Schoenfeld residuals; neither method indicated that the assumption of proportional hazards was violated. Confounders were identified a priori as variables that may be associated with both breast cancer incidence and osteopenia/osteoporosis. Multivariable (MV) models were adjusted for menopausal status (premenopausal, postmenopausal), HRT use (ever, never), BMI $\left(\mathrm{kg} / \mathrm{m}^{2}\right)$, bilateral oophorectomy at age $<45$ years (yes, no), physical activity (MET-h/wk), smoking status (ever, never), and alcohol use (g/d). To account for a small percentage of missing data $(<1 \%$ missing) in covariates, we imputed missing data with the most common category for categorical covariates and the median value for continuous covariates among cancer-free women.

To identify whether bone loss differed by subgroups of breast cancer survivors, we examined the risk of osteopenia and osteoporosis in survivors stratified by age at diagnosis, menopausal status at diagnosis, ER tumor status, and breast cancer treatment relative to cancer-free women. For models that stratified breast cancer survivors by ER status, survivors were restricted to invasive breast cancer because ER status was not routinely measured in women with a stage 0 diagnosis. We were unable to conduct analyses by HER2 status or triple-negative breast cancer, due to small numbers. We additionally conducted analyses by family history of breast cancer (no family history, first-degree relative only, first- and second-degree relatives) and an exploratory analysis by $B R C A 1 / 2$ carrier status among a subgroup of women with genetic testing.

Finally, to examine whether risk of osteopenia and osteoporosis varied by time since diagnosis, we used time since enrollment as the time metric and restricted survivors to women diagnosed with breast cancer within 1 year prior to enrollment. Models were then stratified by follow-up time ( $\leq 2$ years and $>2$ years), and heterogeneity was tested using the likelihood ratio test.

Analyses were conducted using SAS version 9.4 (SAS Institute Inc., Cary, NC, USA) and Stata version 14.0 (StataCorp LP, College Station, TX, USA) software. All statistical tests were two-sided, and $p$ values $\leq 0.05$ were considered statistically significant.

\section{Results}

Age and age-adjusted baseline characteristics were compared in breast cancer survivors and cancer-free women (Table 1). Compared with cancer-free women, breast cancer survivors were more likely to be slightly older, postmenopausal, and current vitamin D users and less likely to have had a bilateral oophorectomy at a young age and a family history of breast cancer. Both survivors and cancer-free women were predominately white and highly educated ( $\geq 4$ years of college). Among breast cancer survivors, the mean time from diagnosis to enrollment was 1.4 years, and the mean age at diagnosis was 47 years. Over $80 \%$ of breast cancer survivors were diagnosed with a first invasive breast cancer, and $76 \%$ had ER-positive breast tumors. In addition, all breast cancer survivors received surgery prior to adjuvant therapy; $65 \%$ of survivors received hormone therapy $(67 \%$ tamoxifen, $41 \%$ aromatase inhibitors); and $50 \%$ of survivors received chemotherapy.

During an average of 5.8 years of follow-up, $66 \%$ of breast cancer survivors and $53 \%$ of cancer-free women reported having a bone density examination, and there were 112 incident cases of osteopenia and/or osteoporosis (75\% osteopenia only). The incidence rates for osteopenia and osteoporosis were 44 cases/1000 person-years among breast cancer survivors and 19 cases/1000 person-years in their cancer-free peers. Overall, breast cancer survivors had a $68 \%$ higher risk of osteopenia and osteoporosis than cancer-free women (MV-HR $=1.68,95 \% \mathrm{CI}=1.12-2.50)$ (Table 2). Results were similar when we restricted our analytic population to women who reported having a bone density examination prior to baseline and during followup $(\mathrm{MV}-\mathrm{HR}=1.90,95 \% \mathrm{CI}=1.08-3.34 ; \mathrm{MV}-\mathrm{HR}=1.72$, $95 \% \mathrm{CI}=1.14-2.58$, respectively). The results were also 
Table 1 Age and age-adjusted baseline characteristics of cancer-free women and breast cancer survivors in the BOSS cohort study

\begin{tabular}{|c|c|c|c|}
\hline \multirow[t]{2}{*}{ Characteristic } & \multirow{2}{*}{$\begin{array}{l}\text { Cancer-free } \\
(n=567)\end{array}$} & \multirow{2}{*}{$\begin{array}{l}\text { Survivors }^{a} \\
(n=211)\end{array}$} & \multirow[t]{2}{*}{$p$ Value } \\
\hline & & & \\
\hline Age $^{\mathrm{b}}$, years, mean $(S D)$ & $44.7(11.3)$ & $48.1(10.3)$ & $<0.001$ \\
\hline Race, white, \% & 88.7 & 83.3 & 0.02 \\
\hline Education, $\geq 4$ years of college, $\%$ & 77.6 & 77.4 & 0.72 \\
\hline Postmenopausal, \% & 27.4 & 51.6 & $<0.001$ \\
\hline Age at menopause ${ }^{c}$, years, mean (SD) & $49.6(4.9)$ & $48.8(3.4)$ & 0.86 \\
\hline Bilateral oophorectomy at age $<45$ years ${ }^{d}, \%$ & 49.0 & 34.3 & 0.02 \\
\hline $\mathrm{BMI}, \mathrm{kg} / \mathrm{m}^{2}$, mean (SD) & $26.2(5.1)$ & $25.9(3.1)$ & 0.29 \\
\hline Physical activity, MET-h/wk', mean (SD) & $29.4(27.9)$ & $26.0(15.5)$ & 0.29 \\
\hline Alcohol intake, g/d, mean (SD) & $5.7(7.5)$ & $5.7(4.8)$ & 0.99 \\
\hline \multicolumn{4}{|l|}{ Smoking status, \% } \\
\hline Never & 58.8 & 52.5 & \multirow[t]{4}{*}{0.55} \\
\hline Former & 36.7 & 44.8 & \\
\hline Current & 4.2 & 2.7 & \\
\hline Missing & 0.3 & 0.0 & \\
\hline HRT ever use, \% & 15.1 & 14.5 & 0.04 \\
\hline Ever mammogram ${ }^{f}, \%$ & 99.5 & 97.8 & 0.63 \\
\hline Ever pap smear, \% & 98.6 & 99.1 & 0.15 \\
\hline Current vitamin D supplement use, \% & 7.8 & 20.8 & $<0.001$ \\
\hline Current calcium supplement use, $\%$ & 25.5 & 28.1 & 0.97 \\
\hline Bone density examination, \% & 28.9 & 43.0 & 0.02 \\
\hline Bone density examination in women aged $\geq 45$ years, $\%$ & 51.2 & 60.0 & 0.08 \\
\hline Ever broken bone, \% & 6.4 & 6.8 & 0.84 \\
\hline \multicolumn{4}{|l|}{ Family history of breast cancer, $\%$} \\
\hline No family history & 14.7 & 38.9 & \multirow[t]{4}{*}{$<0.001$} \\
\hline First-degree relative only & 64.8 & 50.7 & \\
\hline First- and second-degree relatives & 17.0 & 9.0 & \\
\hline Missing & 3.5 & 1.4 & \\
\hline \multicolumn{4}{|l|}{ BRCA1/2 status ${ }^{9}, \%$} \\
\hline Negative & 69.7 & 73.9 & \multirow[t]{3}{*}{0.33} \\
\hline Positive & 27.3 & 19.4 & \\
\hline Variants of uncertain significance & 2.9 & 6.7 & \\
\hline Age at diagnosis, years, mean (SD) & - & $46.8(10.2)$ & - \\
\hline Time from diagnosis to baseline, years, mean (SD) & - & $1.4(1.3)$ & - \\
\hline Invasive breast cancer (stage I-|II), \% & - & 82.5 & - \\
\hline Estrogen receptor status ${ }^{\mathrm{h}}, \%$ & - & & - \\
\hline Positive & - & 75.9 & - \\
\hline Negative & - & 23.6 & - \\
\hline Missing/untested & - & $<1.0$ & - \\
\hline HER2 status ${ }^{\mathrm{h}}, \%$ & - & & - \\
\hline Positive & - & 14.4 & - \\
\hline Negative & - & 81.6 & - \\
\hline Missing/untested & - & 3.5 & - \\
\hline Triple-negative status ${ }^{\mathrm{h}}, \%$ & - & 19.0 & - \\
\hline
\end{tabular}


Table 1 Age and age-adjusted baseline characteristics of cancer-free women and breast cancer survivors in the BOSS cohort study (Continued)

\begin{tabular}{|c|c|c|c|}
\hline \multirow[t]{2}{*}{ Characteristic } & Cancer-free & Survivors $^{\mathrm{a}}$ & \multirow[t]{2}{*}{$p$ Value } \\
\hline & $(n=567)$ & $(n=211)$ & \\
\hline Breast cancer treatment ${ }^{i, j}, \%$ & - & & - \\
\hline Surgery & - & 100.0 & - \\
\hline Chemotherapy & - & 49.8 & - \\
\hline Hormone therapy, any & - & 65.4 & - \\
\hline Hormone therapy, by type ${ }^{k}$ & - & & - \\
\hline Tamoxifen & - & 67.4 & - \\
\hline Aromatase inhibitor & - & 41.3 & - \\
\hline
\end{tabular}

Abbreviations: BMI Body mass index, HER2 Human epidermal growth factor receptor 2, HRT Hormone replacement therapy

Values are means (SD) or percentages and are standardized to the age distribution of the study population

${ }^{a}$ Women were diagnosed with stages 0 -III breast cancer $\leq 5$ years prior to baseline

${ }^{b}$ Value is not age-adjusted

${ }^{c}$ Among postmenopausal women

${ }^{d}$ Among women who had both ovaries removed $(n=86)$

${ }^{\mathrm{A}}$ Metabolic equivalents from recreational and occupational activity

${ }^{f}$ Among women aged $\geq 50$ years

${ }_{9}^{9}$ Among women tested for BRCA status $(n=414)$

${ }^{\mathrm{h}}$ Among invasive cases only $(n=174)$

'Treatment groups are not mutually exclusive

${ }^{\mathrm{j}}$ Chemotherapy and hormone therapy are adjuvant

${ }^{\mathrm{k}}$ Seven percent of breast cancer survivors received both tamoxifen and aromatase inhibitors $(n=15)$

Table 2 Risk of incident osteopenia and osteoporosis among breast cancer survivors compared with cancer-free women

\begin{tabular}{|c|c|c|c|}
\hline & Events/person-years & Age-adjusted HR (95\% Cl) & MV-adjusted HR $(95 \% \mathrm{Cl})^{\mathrm{a}}$ \\
\hline \multicolumn{4}{|l|}{ Overall } \\
\hline Cancer-free & $67 / 3509$ & 1.00 (reference) & 1.00 (reference) \\
\hline Breast cancer survivors & $45 / 1026$ & $2.01(1.38-2.94)$ & $1.68(1.12-2.50)$ \\
\hline \multicolumn{4}{|c|}{ Excluding women without bone density examinations prior to baseline } \\
\hline Cancer-free & $27 / 1023$ & 1.00 (reference) & 1.00 (reference) \\
\hline Breast cancer survivors & $27 / 497$ & $1.96(1.15-3.36)$ & $1.90(1.08-3.34)$ \\
\hline \multicolumn{4}{|c|}{ Excluding women without bone density examinations during follow-up } \\
\hline Cancer-free & $63 / 1890$ & 1.00 (reference) & 1.00 (reference) \\
\hline Breast cancer survivors & $45 / 703$ & $1.89(1.29-2.78)$ & $1.72(1.14-2.58)$ \\
\hline \multicolumn{4}{|c|}{ Excluding early bilateral oophorectomy prior to baseline ${ }^{b}$} \\
\hline Cancer-free & $64 / 3347$ & 1.00 (reference) & 1.00 (reference) \\
\hline Breast cancer survivors & $42 / 957$ & $1.93(1.30-2.85)$ & $1.63(1.08-2.46)$ \\
\hline \multicolumn{4}{|c|}{ Excluding pre- to postmenopausal during follow-up } \\
\hline Cancer-free & $34 / 2308$ & 1.00 (reference) & 1.00 (reference) \\
\hline Breast cancer survivors & $32 / 745$ & $2.18(1.34-3.55)$ & $1.57(0.93-2.63)$ \\
\hline \multicolumn{4}{|c|}{ Excluding current vitamin $D$ users ${ }^{c}$} \\
\hline Cancer-free & $60 / 3263$ & 1.00 (reference) & 1.00 (reference) \\
\hline Breast cancer survivors & $36 / 820$ & $2.03(1.34-3.08)$ & $1.68(1.08-2.61)$ \\
\hline \multicolumn{4}{|c|}{ Excluding current calcium users ${ }^{c}$} \\
\hline Cancer-free & $40 / 2637$ & 1.00 (reference) & 1.00 (reference) \\
\hline Breast cancer survivors & $28 / 743$ & $2.14(1.32-3.48)$ & $1.59(0.95-2.68)$ \\
\hline
\end{tabular}

Abbreviations: MV Multivariable

${ }^{a}$ Adjusted for age (years), menopausal status (premenopausal, postmenopausal), bilateral oophorectomy at age $<45$ years (yes, no), body mass index (kg/m ${ }^{2}$ ),

physical activity (MET-h/wk), smoking status (never, ever), alcohol intake (g/d), and hormone replacement therapy (never, ever)

${ }^{b}$ Both ovaries removed prior to age 45 years

${ }^{\mathrm{C}}$ Vitamin $\mathrm{D}$ and calcium supplement use was ascertained at baseline 
similar when we excluded women who had premature menopause secondary to a bilateral oophorectomy at age $<45$ years $(\mathrm{MV}-\mathrm{HR}=1.63,95 \% \mathrm{CI}=1.08-2.46)$ and only slightly attenuated when we restricted our analysis to women with no change in menopausal status during follow-up (MV-HR $=1.57,95 \% \mathrm{CI}=0.93-2.63$ ). Finally, the results did not change when we restricted our analytic sample to women without current vitamin D use at baseline $(\mathrm{MV}-\mathrm{HR}=1.68,95 \% \mathrm{CI}=1.08-2.61)$ and became slightly attenuated among women without current calcium use at baseline (MV-HR $=1.59,95 \% \mathrm{CI}=0.95-2.68$ ).

The risk of osteopenia and osteoporosis in breast cancer survivors stratified by age at diagnosis, menopausal status at diagnosis, and ER status was compared with that of cancer-free women (Table 3). Breast cancer survivors diagnosed at age $\leq 50$ years had an almost twofold increased risk of osteopenia and osteoporosis compared with cancer-free women (MV-HR $=1.98$, 95\% $\mathrm{CI}=1.21-3.24)$. Surprisingly, the association was not significant in older women. In addition, breast cancer survivors who were premenopausal at diagnosis had increased risk of osteopenia and osteoporosis relative to their cancer-free peers (MV-HR $=1.76,95 \% \mathrm{CI}=1.09$ 2.84 ), and this risk was similar but attenuated among women who were postmenopausal at diagnosis (MV-HR = $1.58,95 \% \mathrm{CI}=0.86-2.89$ ). Finally, women with ER-positive tumors had an over twofold increased risk of osteopenia and osteoporosis compared with cancer-free women $(\mathrm{MV}-\mathrm{HR}=2.10 ; 95 \%=1.34-3.29)$. Although women with ER-negative tumors had a modest increased risk of osteopenia and osteoporosis relative to their cancer-free peers, the association was not statistically significant $(\mathrm{MV}-\mathrm{HR}=1.26$;
95\% CI $=0.54-2.94)$. Results were attenuated but did not differ by family history of breast cancer and $B R C A 1 / 2$ carrier status (data not shown).

Next, the risk of osteopenia and osteoporosis in breast cancer survivors stratified by treatment compared with cancer-free women was examined (Fig. 1). Breast cancer survivors treated with chemotherapy plus hormone therapy had an over twofold increased risk of osteopenia and osteoporosis compared with cancer-free women $(\mathrm{MV}-\mathrm{HR}=2.70 ; \quad 95 \% \mathrm{CI}=1.56-4.68)$. No significant association was observed for breast cancer survivors treated with surgery, chemotherapy, or hormone therapy alone compared with cancer-free women. Breast cancer survivors treated with aromatase inhibitors alone and combined chemotherapy plus aromatase inhibitors had a greater than two- and threefold increased risk of osteopenia and osteoporosis compared with cancer-free women $(\mathrm{MV}-\mathrm{HR}=2.72,95 \% \mathrm{CI}=1.31-5.65$; $\mathrm{MV}-\mathrm{HR}=3.83, \quad 95 \% \mathrm{CI}=1.87-7.83$, respectively). In addition, breast cancer survivors treated with chemotherapy plus tamoxifen had a greater than twofold increased risk compared with cancer-free women $(\mathrm{MV}-\mathrm{HR}=2.48$, 95\% CI $=1.16-5.30$ ).

Finally, breast cancer survivors diagnosed within 1 year prior to enrollment had a greater than twofold increased risk of osteopenia and osteoporosis compared to their cancer-free peers within the first 2 years of follow-up $(\mathrm{MV}-\mathrm{HR}=2.74,95 \% \mathrm{CI}=1.37-5.47)$ and a nonsignificant $85 \%$ increased risk of osteopenia and osteoporosis after 2 years of follow-up (MV-HR $=1.85,95 \%$ $\mathrm{CI}=0.98-3.51)$, although the $p$ value for heterogeneity was not significant $(p=0.44)$ (Table 4$)$.

Table 3 Risk of incident osteopenia and osteoporosis among breast cancer survivors compared with cancer-free women, stratified by characteristics at diagnosis

\begin{tabular}{|c|c|c|c|}
\hline & Events/person-years & Age-adjusted HR (95\% Cl) & MV-adjusted HR $(95 \% \mathrm{Cl})^{\mathrm{a}}$ \\
\hline \multicolumn{4}{|l|}{ Age at diagnosis } \\
\hline Cancer-free & $67 / 3509$ & 1.00 (reference) & 1.00 (reference) \\
\hline$\leq 50$ years & $27 / 651$ & $2.34(1.46-3.75)$ & $1.98(1.21-3.24)$ \\
\hline$>50$ years & $18 / 375$ & $1.64(0.93-2.88)$ & $1.34(0.75-2.40)$ \\
\hline \multicolumn{4}{|l|}{ Menopausal status at diagnosis } \\
\hline Cancer-free & $67 / 3509$ & 1.00 (reference) & 1.00 (reference) \\
\hline Premenopausal at diagnosis & $27 / 728$ & $1.97(1.24-3.12)$ & $1.76(1.09-2.84)$ \\
\hline Postmenopausal at diagnosis & $18 / 298$ & $2.05(1.15-3.64)$ & $1.58(0.86-2.89)$ \\
\hline \multicolumn{4}{|l|}{ ER status $^{\mathrm{b}}$} \\
\hline Cancer-free & $67 / 3509$ & 1.00 (reference) & 1.00 (reference) \\
\hline ER-negative & $7 / 200$ & $1.68(0.76-3.72)$ & $1.26(0.54-2.94)$ \\
\hline ER-positive & $32 / 611$ & $2.32(1.52-3.55)$ & $2.10(1.34-3.29)$ \\
\hline
\end{tabular}

Abbreviations: $E R$ Estrogen receptor, $M V$ Multivariable

${ }^{a}$ Adjusted for age (years), menopausal status (premenopausal, postmenopausal), bilateral oophorectomy at age $<45$ years (yes, no), body mass index (kg/ $\mathrm{m}^{2}$ ), physical activity (MET-h/wk), smoking status (never, ever), alcohol intake (g/d), and hormone replacement therapy (never, ever)

${ }^{\mathrm{b}}$ Breast cancer survivors were restricted to stages I-III 


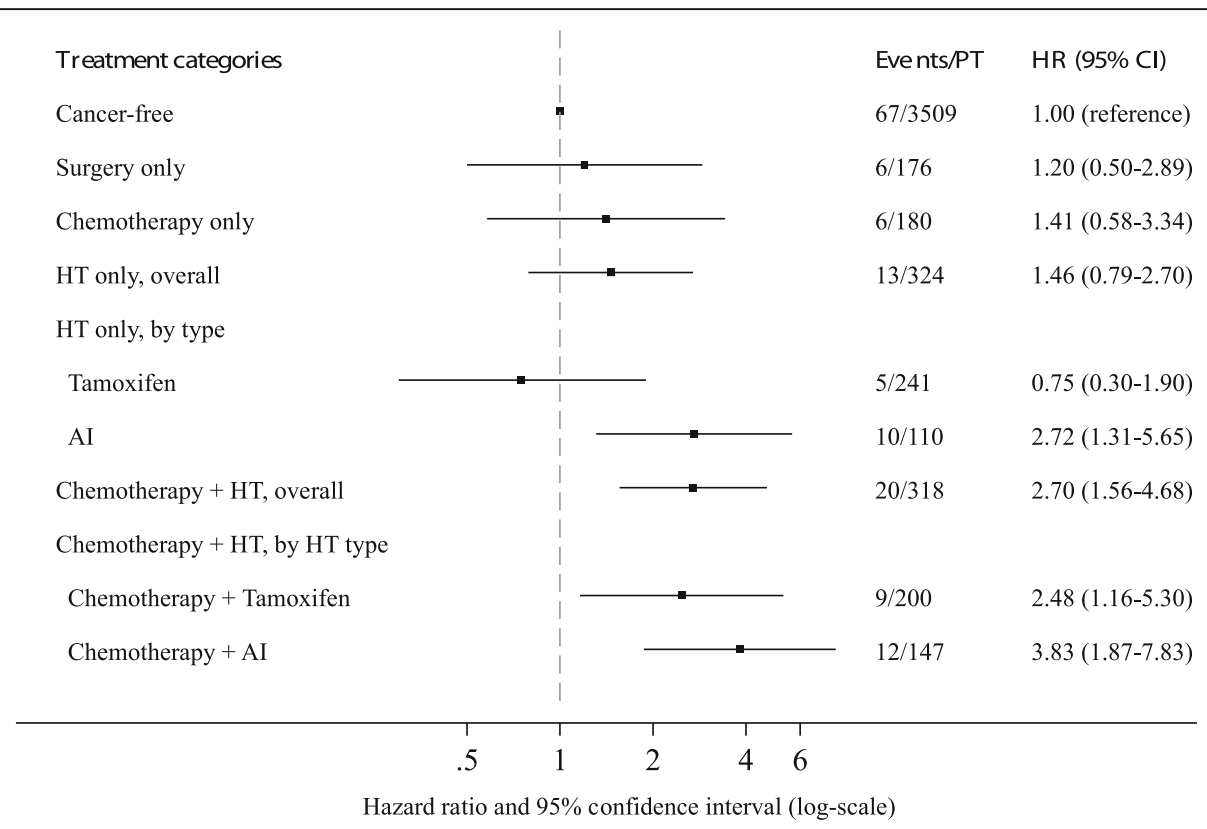

Fig. 1 Abbreviations: Multivariable HRs (95\% Cls) for incident osteopenia and osteoporosis among breast cancer survivors, stratified by type of treatment, compared with cancer-free women. Models are adjusted for Data are adjusted for age (years), menopausal status (premenopausal, postmenopausal), bilateral oophorectomy at age < 45 years (yes, no), body mass index $\left(\mathrm{kg} / \mathrm{m}^{2}\right)$, physical activity (MET-h/wk), smoking status (never, ever), alcohol intake (g/d), and hormone replacement therapy (never, ever). Abbreviations: Al Aromatase inhibitor, HT Hormone therapy, PT Person time in years

\section{Discussion}

To our knowledge, this is the first study to prospectively assess risk of osteopenia and osteoporosis in young and recently diagnosed breast cancer survivors compared with their cancer-free peers in a familial high-risk cohort. In this prospective study, the incidence of osteopenia and osteoporosis was almost twofold higher in breast cancer survivors than in cancer-free women over an average of 5.8 years of follow-up. The results were also similar when we excluded women with premature menopause, suggesting an effect of cancer treatment on bone health that is independent of early menopause. Breast cancer survivors who were younger, had ER-positive tumors, received aromatase inhibitors alone, or received combined chemotherapy with aromatase inhibitors or tamoxifen had a higher risk of osteopenia and osteoporosis than cancerfree women. Importantly, this was after accounting for age, menopause, and other risk factors for bone loss.

The majority of prior studies have examined bone health in breast cancer survivors without a cancer-free

Table 4 Risk of incident osteopenia and osteoporosis among recent breast cancer survivors compared with cancer-free women, stratified by follow-up time ${ }^{a}$

\begin{tabular}{llll}
\hline & Events/person-years & Age-adjusted HR (95\% Cl) & MV-adjusted HR (95\% Cl) \\
\hline $\begin{array}{l}\text { Overall } \\
\text { Cancer-free }\end{array}$ & $67 / 3497$ & 1.00 (reference) & 1.00 (reference) \\
$\begin{array}{l}\text { Survivors } \\
\text {-2 years }\end{array}$ & $27 / 475$ & $2.49(1.58-3.91)$ & $2.17(1.37-3.46)$ \\
$\quad$ Cancer-free & $22 / 1126$ & 1.00 (reference) & 1.00 (reference) \\
$\quad$ Survivors & $14 / 214$ & $3.15(1.61-6.17)$ & $2.74(1.37-5.47)$ \\
$\begin{array}{l}\text { 3+ years } \\
\text { Cancer-free }\end{array}$ & & 1.00 (reference) \\
$\quad$ Survivors & $45 / 2371$ & 1.00 (reference) & $1.85(0.98-3.51)$ \\
\hline
\end{tabular}

\footnotetext{
Abbreviations: MV Multivariable
}

${ }^{\mathrm{a} B r e a s t}$ cancer survivors were restricted to women diagnosed within 1 year prior to enrollment

${ }^{b}$ Adjusted for age (years), menopausal status (premenopausal, postmenopausal), bilateral oophorectomy at age $<45$ years (yes, no), body mass index (kg/ $\mathrm{m}^{2}$ ), physical activity (MET-h/wk), smoking status (never, ever), alcohol intake (g/d), and hormone replacement therapy (never, ever)

$p(\mathrm{~s})$ for the likelihood ratio test of the interaction between breast cancer status and time was 0.42 for age- and 0.44 for $M V$-adjusted models 
comparison group [12-20]. Several studies have found a higher risk of fracture in women diagnosed with breast cancer than in cancer-free women [21-23]; however, results have been inconsistent $[24,25]$. Even fewer epidemiologic studies have assessed osteopenia and osteoporosis risk in women with breast cancer compared with their cancer-free peers within the same cohort [8-10]. Furthermore, these studies have included primarily older and long-term survivors. The first of these studies was conducted in the Women's Health Initiative Observational Study (WHI-OS). This study compared the prevalence of osteoporosis and the rate of bone loss in postmenopausal breast cancer survivors compared with cancer-free women [8]. Although the investigators found that breast cancer survivors had a higher prevalence of low bone density and osteoporosis at baseline, they did not have an increased rate of bone loss compared with cancer-free women over follow-up. However, breast cancer survivors were identified from prevalent cases at study enrollment, and the time from breast cancer diagnosis to study enrollment was not reported. Therefore, it is possible that the rate of bone loss may have been assessed to late after cancer diagnosis or treatment cessation, particularly if substantial bone loss occurred shortly after diagnosis or treatment.

The second study was a retrospective registry study in the Cancer Genetics Network conducted to assess early and late effects of cancer treatment [9]. In this study, the authors assessed osteopenia and osteoporosis risk based on self-report in women with and without invasive breast cancer and found a significant positive association for both outcomes ( $\mathrm{HR}=2.1,95 \% \mathrm{CI}=1.8-2.4$ for osteopenia; $\mathrm{HR}=1.5,95 \% \mathrm{CI}=1.2-1.9$ for osteoporosis). Although this study included younger women with familial cancer risk, breast cancer survivors were identified from 1990 to 2009, and history of bone health was collected retrospectively in 2009. Among these breast cancer survivors, over $70 \%$ were diagnosed $\geq 10$ years prior to the assessment of self-reported bone health in 2009, and thus the study was susceptible to substantial recall bias.

The third study was conducted among the U.K. General Practice Research Database to examine long-term health outcomes among older cancer survivors and cancer-free individuals (overall mean age $=66.9$ years, $\mathrm{SD}=12.3$ years) [10]. The authors assessed osteoporosis risk, but not osteopenia, based on medical records among breast cancer survivors and found that survivors had a $26 \%$ higher risk of osteoporosis than cancer-free women ( $H R=1.26,95 \%$ $\mathrm{CI}=1.13-1.40)$. None of these studies have assessed these associations by tumor subtype or incorporated detailed information on cancer treatment and bone density examination history. In addition, only one study has previously assessed osteopenia risk [9], an earlier indication of bone loss, which is also associated with a high fracture risk.
The most common cause of bone loss in women is menopause and aging. Aging is associated with greater bone resorption and less bone formation, whereas menopause induces accelerated bone loss due to lowering levels of endogenous estrogen [26]. Therefore, a cancer-free comparison of similar age and menopausal status is important when assessing bone loss. Given that we still observed significantly higher bone loss in breast cancer survivors relative to their cancer-free peers after accounting for age and menopause, it is likely that the additional bone loss is due to the effect of treatment on bone formation.

We observed a greater than twofold increased risk of osteopenia and osteoporosis in women diagnosed with ER-positive tumors, which is likely due to hormone therapy rather than to differences in tumor biology. This is supported by the fact that the highest risk of osteopenia and osteoporosis was found among breast cancer survivors treated with aromatase inhibitors alone and chemotherapy plus aromatase inhibitors. These findings are in agreement with the underlying biology of aromatase inhibitors [27], as well as with studies in breast cancer survivors [16-20] and high-risk women in chemoprevention trials $[28,29]$. Aromatase inhibitors, prescribed to postmenopausal women with ER-positive tumors, blocks the aromatase enzyme, resulting in a hypoestrogenic state associated with bone loss [27]. We found no association among women with tamoxifen use alone, a group that was primarily premenopausal at baseline (mean age at baseline $=46$ years; $76 \%$ premenopausal at baseline). However, we did observe an almost twofold increased risk of osteopenia and osteoporosis among women with chemotherapy plus tamoxifen use (mean age at baseline $=43 ; 50 \%$ premenopausal at baseline). Although tamoxifen, a selective ER modulator, is generally thought to be protective against bone loss in postmenopausal women [30], reports suggest that it may cause bone loss among premenopausal women due to premature menopause $[13,31]$. Chemotherapy may also cause bone loss due to treament-induced premature menopause in premenopausal women [32] and may have direct toxic effects on bone formation cells [27]. In addition, medications commonly presribed along with chemotherapy (e.g., corticosteroids) have also been associated with bone loss [33]. Therefore, it is biologically plausible that chemotherapy plus hormone therapy might have a joint deleterious effect on bone health early in treatment.

The strengths of this study include the prospective study design, direct comparison with cancer-free women from the same cohort, and detailed information on cancer treatment. There are also several limitations to our analysis. First, our sample size may have limited our power to detect small to moderate associations. Second, osteopenia and osteoporosis incidence was ascertained on the basis of self-reported physician diagnosis and may be susceptible to misclassification. However, $96 \%$ of women who 
reported a diagnosis of osteopenia or osteoporosis also reported receiving a bone density examination. Third, breast cancer survivors may have increased surveillance for bone health and therefore may be more likely than cancer-free women to be diagnosed with osteopenia and osteoporosis. In our cohort, breast cancer survivors were slightly more likely than cancer-free women to have had a bone density examination at baseline ( $43 \%$ vs. $29 \% ; 60 \%$ vs. $51 \%$ among women aged $\geq 45$ years) and in follow-up (66\% vs. $53 \%$ ). However, sensitivity analyses to further reduce the possibility of undetected prevalent or incident cases found that results were similar when restricted to women with bone density examinations prior to baseline $(\mathrm{MV}-\mathrm{HR}=1.90$, 95\% $\mathrm{CI}=1.08-3.34)$ and during follow-up (MV-HR = 1.72 , 95\% CI $=1.14-2.58$ ). Furthermore, both women with and without breast cancer in our cohort underwent close monitoring for their health. Specifically, overall health screening history at baseline was similar in breast cancer survivors compared with cancer-free women (e.g., 99\% vs. $99 \%$ had ever had a pap smear; $100 \%$ vs. $98 \%$ had ever had a mammogram among women aged $\geq 50$ years). Finally, our results may not be generalizable to other populations, because our study population was composed predominately of white and highly educated women at high risk for breast cancer. However, we believe that the underlying biology of cancer treatment and its effect on bone health are likely similar across ethnicities. The homogeneity of our study population also improves the internal validity of this study because it reduces the influence of potential unmeasured factors.

\section{Conclusions}

In summary, our results demonstrate that incident osteopenia and osteoporosis are significantly higher in young breast cancer survivors within a few years of diagnosis than in cancer-free women and that risk varies by cancer treatment. These findings provide support for a baseline evaluation of bone density and fracture risk assessment close to breast cancer diagnosis, particularly among young survivors being treated with combined chemotherapy and hormone therapy, so that prevention strategies and appropriate monitoring can be implemented early. Future studies are needed to address the frequency of monitoring in breast cancer survivors by specific age and treatment groups.

\section{Abbreviations \\ BMI: Body mass index; BOSS: Breast and Ovarian Surveillance Service; ER: Estrogen receptor; HER2: Human epidural growth factor receptor 2; HRT: Hormone replacement therapy; METs: Metabolic equivalents of tasks}

\section{Acknowledgements}

The authors thank the State of Maryland, the Maryland Cigarette Restitution Fund, and the National Program of Cancer Registries of the Centers for Disease Control and Prevention for the funds that helped support the collection and availability of the cancer registry data. The Maryland Cancer Registry contributed cancer incidence data to this project. Center for Cancer Prevention and Control,
Department of Health and Mental Hygiene, 201 West Preston Street, Room 400, Baltimore, MD 21201; https://phpa.health.maryland.gov/cancer/Pages/ mcr_home.aspx, 410-767-4055. The authors also thank the participants and staff of the BOSS cohort study.

\section{Funding}

This research was supported in part by the Breast Cancer Research Foundation, the Avon Breast Cancer Research Program Network, and grants from the National Cancer Institute at the National Institutes of Health (T32-CA009314, P50CA098252, and P30CA06973).

\section{Availability of data and materials}

The datasets used in the current study may be made available upon application to the BOSS study (Principal Investigator Kala Visvanathan).

\section{Authors' contributions}

This analysis was conceived by $C R$ and KV. CR and KV contributed to the design and interpretation of data. Acquisition of data was performed by BJM $\mathrm{MMO}, \mathrm{BCH}$, and MSM, Data analysis was performed by CR and KV. CR, BJM, $\mathrm{BSRR}, \mathrm{MMO}, \mathrm{BCH}, \mathrm{MSM}, \mathrm{DP}, \mathrm{DKA}$, and $\mathrm{KV}$ were involved in critically revising the manuscript for intellectual content. All authors read and approved the final manuscript for publication.

\section{Ethics approval and consent to participate}

Written informed consent was obtained for all subjects, and institutional review board approval was obtained from the Institutional Review Board of Johns Hopkins University.

Consent for publication

Not applicable.

\section{Competing interests}

The authors declare that they have no competing interests.

\section{Publisher's Note}

Springer Nature remains neutral with regard to jurisdictional claims in published maps and institutional affiliations.

\section{Author details}

${ }^{1}$ Department of Epidemiology, Johns Hopkins Bloomberg School of Public Health, Baltimore, MD 21205, USA. ${ }^{2}$ Johns Hopkins Sidney Kimmel Comprehensive Cancer Center, Baltimore, MD, USA. ${ }^{3}$ The Johns Hopkins School of Medicine, Baltimore, MD, USA.

Received: 14 May 2018 Accepted: 12 October 2018

Published online: 13 November 2018

\section{References}

1. Runowicz CD, Leach CR, Henry NL, Henry KS, Mackey HT, Cowens-Alvarado $\mathrm{RL}$, et al. American Cancer Society/American Society of Clinical Oncology breast cancer survivorship care guideline. J Clin Oncol. 2016;34:611-35.

2. Office of the Surgeon General. Bone health and osteoporosis: a report of the Surgeon General. Rockville: U.S. Department of Health and Human Services; 2004.

3. Coleman R, Body JJ, Aapro M, Hadji P, Herrstedt J. Bone health in cancer patients: ESMO Clinical Practice Guidelines. Ann Oncol. 2014;25(Suppl 3): iiii24-37.

4. Gralow JR, Biermann JS, Farooki A, Fornier MN, Gagel RF, Kumar R, et al. NCCN Task Force Report: bone health in cancer care. J Natl Compr Cancer Netw. 2013;11(Suppl 3):S1-50.

5. Wright NC, Looker AC, Saag KG, Curtis JR, Delzell ES, Randall S, et al. The recent prevalence of osteoporosis and low bone mass in the United States based on bone mineral density at the femoral neck or lumbar spine. J Bone Miner Res. 2014:29:2520-6.

6. U.S. Preventive Services Task Force. Screening for osteoporosis: U.S. Preventive Services Task Force recommendation statement. Ann Intern Med. 2011;154:356-64.

7. Karaguzel G, Holick MF. Diagnosis and treatment of osteopenia. Rev Endocr Metab Disord. 2010;11:237-51. 
8. Chen Z, Maricic M, Pettinger M, Ritenbaugh C, Lopez AM, Barad DH, et al. Osteoporosis and rate of bone loss among postmenopausal survivors of breast cancer. Cancer. 2005;104:1520-30.

9. Hill DA, Horick NK, Isaacs C, Domchek SM, Tomlinson GE, Lowery JT, et al. Long-term risk of medical conditions associated with breast cancer treatment. Breast Cancer Res Treat. 2014;145:233-43.

10. Khan NF, Mant D, Carpenter L, Forman D, Rose PW. Long-term health outcomes in a British cohort of breast, colorectal and prostate cancer survivors: a database study. Br J Cancer. 2011;105 Suppl 1:S29-37.

11. Gross AL, May BJ, Axilbund JE, Armstrong DK, Roden RBS, Visvanathan K. Weight change in breast cancer survivors compared to cancer-free women: a prospective study in women at familial risk of breast cancer. Cancer Epidemiol Biomark Prev. 2015;24:1262-9.

12. Love RR, Mazess RB, Barden HS, Epstein S, Newcomb PA, Jordan VC, et al. Effects of tamoxifen on bone mineral density in postmenopausal women with breast cancer. N Engl J Med. 1992;326:852-6.

13. Powles TJ, Hickish T, Kanis JA, Tidy A, Ashley S. Effect of tamoxifen on bone mineral density measured by dual-energy $x$-ray absorptiometry in healthy premenopausal and postmenopausal women. J Clin Oncol. 1996;14:78-84.

14. Sverrisdóttir Á, Fornander T, Jacobsson H, Schoultz EV, Rutqvist LE. Bone mineral density among premenopausal women with early breast cancer in a randomized trial of adjuvant endocrine therapy. J Clin Oncol. 2004;22: 3694-9.

15. Zaman K, Thürlimann B, Huober J, Schönenberger A, Pagani O, Lüthi J, Simcock $\mathrm{M}$, et al. Bone mineral density in breast cancer patients treated with adjuvant letrozole, tamoxifen, or sequences of letrozole and tamoxifen in the BIG 1-98 study. Ann Oncol. 2012;23:1474-81.

16. Coleman RE, Banks LM, Girgis SI, Kilburn LS, Vrdoljak E, Fox J, et al. Skeletal effects of exemestane on bone-mineral density, bone biomarkers, and fracture incidence in postmenopausal women with early breast cance participating in the Intergroup Exemestane Study (IES): a randomised controlled study. Lancet Oncol. 2007:8:119-27.

17. Cuzick J, Sestak I, Baum M, Buzdar A, Howell A, Dowsett M, et al. Effect of anastrozole and tamoxifen as adjuvant treatment for early-stage breast cancer: 10-year analysis of the ATAC trial. Lancet Oncol. 2010;11:1135-41.

18. Eastell R, Hannon RA, Cuzick J, Dowsett M, Clack G, Adams JE. Effect of an aromatase inhibitor on BMD and bone turnover markers: 2-year results of the Anastrozole, Tamoxifen, Alone or in Combination (ATAC) trial (18233230). J Bone Miner Res. 2006;21:1215-23.

19. Lønning PE, Geisler J, Krag LE, Erikstein B, Bremnes Y, Hagen Al, et al. Effects of exemestane administered for 2 Years versus placebo on bone mineral density, bone biomarkers, and plasma lipids in patients with surgically resected early breast cancer. J Clin Oncol. 2005;23:5126-37.

20. Perez EA, Josse RG, Pritchard KI, Ingle JN, Martino S, Findlay BP, et al. Effect of letrozole versus placebo on bone mineral density in women with primary breast cancer completing 5 or more years of adjuvant tamoxifen: a companion study to NCIC CTG MA.17. J Clin Oncol. 2006;24:3629-35.

21. Chen Z, Maricic M, Aragaki AK, Mouton C, Arendell L, Lopez AM, et al. Fracture risk increases after diagnosis of breast or other cancers in postmenopausal women: results from the Women's Health Initiative. Osteoporos Int. 2009;20:527-36.

22. Chen Z, Maricic M, Bassford TL, Pettinger M, Ritenbaugh C, Lopez AM, et al. Fracture risk among breast cancer survivors: results from the women's health initiative observational study. Arch Intern Med. 2005;165:552-8.

23. Tsa $\mathrm{CH}$, Muo $\mathrm{CH}$, Tzeng HE, Tang $\mathrm{CH}$, Hsu HC, Sung FC. Fracture in asian women with breast cancer occurs at younger age. PLoS One. 2013;8:e75109.

24. Lamont EB, Lauderdale DS. Low risk of hip fracture among elderly breast cancer survivors. Ann Epidemiol. 2003;13:698-703.

25. Pawloski PA, Geiger AM, Haque R, Kamineni A, Fouayzi H, Ogarek J, et al. Fracture risk in older, long-term survivors of early-stage breast cancer. J Am Geriatr Soc. 2013;61:888-95.

26. Demontiero O, Vidal C, Duque G. Aging and bone loss: new insights for the clinician. Ther Adv Musculoskelet Dis. 2012;4:61-76.

27. Taxel $P$, Choksi $P$, Van Poznak C. The management of osteoporosis in breast cancer survivors. Maturitas. 2012;73:275-9.

28. Cheung AM, Tile L, Cardew S, Pruthi S, Robbins J, Tomlinson G, et al. Bone density and structure in healthy postmenopausal women treated with exemestane for the primary prevention of breast cancer: a nested substudy of the MAP.3 randomised controlled trial. Lancet Oncol. 2012;13:275-84.

29. Sestak I, Singh S, Cuzick J, Blake GM, Patel R, Gossiel F, et al. Changes in bone mineral density at 3 years in postmenopausal women receiving anastrozole and risedronate in the IBIS-II bone substudy: an international, double-blind, randomised, placebo-controlled trial. Lancet Oncol. 2014;15: 1460-8.

30. Kristensen B, Ejlertsen B, Dalgaard P, Larsen L, Holmegaard SN, Transbøl I, et al. Tamoxifen and bone metabolism in postmenopausal low-risk breast cancer patients: a randomized study. J Clin Oncol. 1994;12:992-7.

31. Vehmanen L, Elomaa I, Blomqvist C, Saarto T. Tamoxifen treatment after adjuvant chemotherapy has opposite effects on bone mineral density in premenopausal patients depending on menstrual status. J Clin Oncol. 2006; 24:675-80.

32. Shapiro CL, Manola J, Leboff M. Ovarian failure after adjuvant chemotherapy is associated with rapid bone loss in women with early-stage breast cancer. J Clin Oncol. 2001;19:3306-11.

33. Pitts CJD, Kearns AE. Update on medications with adverse skeletal effects. Mayo Clin Proc. 2011;86:338-43.

\section{Ready to submit your research? Choose BMC and benefit from:}

- fast, convenient online submission

- thorough peer review by experienced researchers in your field

- rapid publication on acceptance

- support for research data, including large and complex data types

- gold Open Access which fosters wider collaboration and increased citations

- maximum visibility for your research: over $100 \mathrm{M}$ website views per year

At $\mathrm{BMC}$, research is always in progress.

Learn more biomedcentral.com/submissions 